\title{
Non-invasive epileptic focus localization using EEG-triggered functional MRI and electromagnetic tomography
}

\author{
M. Seeck ${ }^{\mathrm{a}, *}$, F. Lazeyras ${ }^{\mathrm{b}}$, C.M. Michel ${ }^{\mathrm{a}}$, O. Blanke ${ }^{\mathrm{a}}$, C.A. Gericke ${ }^{\mathrm{a}}, \mathrm{J}_{\text {. Ives }}^{\mathrm{c}}, \mathrm{J}_{\text {. Delavelle }}^{\mathrm{b}}$, \\ X. Golay ${ }^{\mathrm{d}}$, C.A. Haenggeli ${ }^{\mathrm{e}}$, N. de Tribolet $^{\mathrm{f}, \mathrm{g}}$, T. Landis ${ }^{\mathrm{a}}$ \\ ${ }^{a}$ Department of Neurology, University Hospital of Geneva, 24 rue Micheli-du-Crest, CH-1211 Geneva 14, Switzerland \\ ${ }^{\mathrm{b}}$ Department of Radiology, University Hospital of Geneva, 24 rue Micheli-du-Crest, CH-1211 Geneva 14, Switzerland \\ ${ }^{\mathrm{c}}$ Department of Neurology, Beth Israel Deaconess Medical Center, Harvard Medical School, Boston, USA \\ ${ }^{\mathrm{d}}$ Institute of Biomedical Engineering and Medical Informatics, University and ETH, Zürich, Switzerland \\ ${ }^{\mathrm{e}}$ Department of Pediatrics, University Hospital of Geneva, 24 rue Micheli-du-Crest, CH-1211 Geneva 14, Switzerland \\ ${ }^{\mathrm{f}}$ Department of Neurosurgery of the University Hospital in Geneva, Geneva, Switzerland \\ ${ }^{\mathrm{g}}$ Department of Neurosurgery of the University Hospital in Lausanne, Lausanne, Switzerland
}

Accepted for publication: 14 January 1998

\begin{abstract}
We present a new approach for non-invasive localization of focal epileptogenic discharges in patients considered for surgical treatment. EEG-triggered functional MR imaging (fMRI) and 3D EEG source localization were combined to map the primary electrical source with high spatial resolution. The method is illustrated by the case of a patient with medically intractable frontal lobe epilepsy. EEG obtained in the MRI system allowed triggering of the fMRI acquisition by the patient's habitual epileptogenic discharges. fMRI revealed multiple areas of signal enhancement. Three-dimensional EEG source localization identified the same active areas and provided evidence of onset in the left frontal lobe. Subsequent electrocorticography from subdural electrodes confirmed spike and seizure onset over this region. This approach, i.e. the combination of EEG-triggered fMRI and 3D EEG source analysis, represents a promising additional tool for presurgical epilepsy evaluation allowing precise non-invasive identification of the epileptic foci. (C) 1998 Elsevier Science Ireland Ltd. All rights reserved
\end{abstract}

Keywords: Functional MRI; EEG; Electromagnetic tomography; Epilepsy; Localization; Surgery

\section{Introduction}

A major goal of the presurgical evaluation of patients suffering from pharmacoresistant epilepsy is the localization of the epileptic focus, preferably with non-invasive techniques. During the past decades, useful tools, such as positron emission tomography (PET) or single-photonemission computer tomography (SPECT) have been developed, which have also contributed to our understanding of the functional anatomy of the brain, but have rarely yielded precise focus localization allowing immediate surgical plan-

\footnotetext{
* Corresponding author. Laboratory of Presurgical Evaluation of Epilepsy, Department of Neurology, University Hospital of Geneva, 24 rue Micheli-du-Crest, CH-1211 Geneva 14, Switzerland. Tel.: +41 22 3728352; fax: +41 22 3728340; e-mail: mase@diogenes.hcuge.ch
}

ning (Marks et al., 1992; Henry et al., 1993; Newton et al., 1995).

Functional magnetic resonance imaging (fMRI) is a relatively new technique, offering a means to study localized neuronal activity on the basis of each individual's brain. Most fMRI studies have been dedicated to the study of stimulus-related functions (e.g. Belliveau et al., 1991). In a few cases, patients with prolonged subclinical or focal seizure activity without major body movements have been studied (Jackson et al., 1994; Detre et al., 1995). fMRI data yielded excellent localizing information of the seizure origin. However, these focal, prolonged non-convulsive seizures without propagation and body movements, represent the optimal situation, but occur rarely in clinical practice. Focal interictal discharges are seen much more frequently and are therefore more likely to be captured. Thus, EEG monitoring during image acquisition is mandatory in order 
to carry out meaningful analysis. A system allowing EEG recording inside the magnet has recently been introduced and preliminary studies showed promising results with regard to technical aspects and focus localization (Ives et al., 1993; Warach et al., 1996). However, as the study of Warach and collaborators showed, multiple areas of signal enhancement can be found, requiring additional detailed temporal analysis in order to identify the leading source.

This temporal information can be derived from EEG source localization methods which are increasingly utilized to determine the area or the laterality of the epileptic focus (Ebersole, 1991; Lantz et al., 1996). New localization algorithms that estimate 3D current density distributions have recently become available (Pascual-Marqui et al., 1994; George et al., 1995). The potential capability of these new methods to characterize the temporal aspects of origin and propagation of the epileptic activity have already been shown (Lantz et al., 1997; Seri et al., 1998).

By combining EEG-triggered fMRI and 3D EEG source localization, the advantages of both techniques (spatial and temporal resolution) could be used for the presurgical evaluation of focal epilepsy, in particular non-lesional extratemporal epilepsy known to be difficult to localize noninvasively. Here we illustrate the investigation of a patient with pharmacoresistant, non-lesional frontal lobe epilepsy.

\section{Materials and methods}

\subsection{Case report}

An 18 year old, right-handed woman suffered from medically-intractable seizures from the age of 7 . Since that age, she presented brief 'absence-like' seizures with staring and manual automatisms which were often followed by a rightsided deviation of the face and mouth, followed by generalized tonic-clonic seizures. No preceding aura was reported. Family history as well as personal history were unrevealing. A seizure frequency of 5-12/week could not be altered by various anticonvulsive treatments. Epilepsy surgery was finally considered and she was referred to our laboratory for long-term video EEG monitoring and complementary examinations.

High resolution MRI did not show a prominent lesion. Nuclear imagery (PET, interictal and ictal SPECT) and neuropsychological testing suggested left-hemispheric or leftfrontal dysfunction without more precise localization. Video and EEG recordings with 29 scalp and two sphenoidal electrodes recorded her habitual seizures and suggested left frontal onset. Her interictal EEG was characterized by frequent, more left than right, frontal spikes.

Invasive monitoring with subdural grids and strips over the lateral frontal, mesial frontal, fronto-orbital, parietal and temporal areas was performed in order to determine more precisely the epileptogenic zone and differentiate it from eloquent or primary motor cortex. Surgery was carried out, based on the findings of invasive monitoring. The analysis of the ictal and interictal discharges allowed us to determine the accuracy of this non-invasive localization technique in comparison with electrocorticography findings.

\section{2. fMRI and EEG acquisition}

After informed consent was given, 16 scalp electrodes were applied according to standard positions of the 10/20 system, referenced to PO4. The EEG equipment to use within the magnet (OptiLink, Neuroscan, Herndon, VA) is described elsewhere (Ives et al., 1993). EEG was recorded continuously on a 64 channel EEG machine (Deltamed SA, France) with a sampling rate of $128 \mathrm{~Hz}$. The blood oxygenation level dependent (BOLD) images were obtained with a 1.5 T Edge system (Picker Int., Cleveland, $\mathrm{OH}$ ) using a multi-shot echo planar imaging (EPI) with the following parameters: echo train length $=8$ echoes, echo time $(\mathrm{TE})=35 \mathrm{~ms}$, repetition time $(\mathrm{TR})=560 \mathrm{~ms}$, number of averages $=1$, field of view $(\mathrm{FOV})=32 \times 16 \mathrm{~cm}^{2}$, matrix size $=128 \times 64$, number of slices $=6$, slice thickness $=6$ $\mathrm{mm}$ with $1 \mathrm{~mm}$ inter-slice distance. The acquisition time was 5.5 s. According to a study of Jiang et al. (1995), after motion correction, a spatial translocation error of not more than $0.23 \mathrm{~mm}$ should be expected, using a gap of $1 \mathrm{~mm}$ between slices.

The EEG monitor was placed next to the MRI console to allow rapid manually-triggered image acquisition. In the first series of images ('activated condition'), acquisition was initiated whenever a discharge was noted on the screen. The mean latency between the appearance of isolated discharges or, if bursts of spikes, the offset of epileptiform discharges, was $0.46 \mathrm{~s}$ (range: $0-5 \mathrm{~s}$ ). To obtain the control condition, $2 \mathrm{mg}$ Clonazepam was slowly given intravenously. Once it was verified that there were no more epileptic discharges, control images were obtained every $12 \mathrm{~s}$. Two series of 60 images each were obtained. The stored EEG was reviewed off-line to reject those sequences that were related to unclear epileptiform EEG signals (e.g. isolated sharp wave). The exclusion of these sequences resulted in 43 triggered multi-slice MRIs and 43 control images, which were then statistically analyzed. The two sets of 43 images were analyzed using a standard crosscorrelation computation of each individual pixel (Bandettini et al., 1993). Bonferroni correction was used to eliminate false positives derived from multiple comparisons. Only pixels showing $P$-values $<0.005$ after correction were considered. Further restriction of the interpretation of the results was applied by considering only those regions where more than 10 neighboring pixels were significantly correlated.

\subsection{Three-dimensional EEG analysis}

The EEG spikes that initiated the fMRI acquisitions were analyzed off-line. A total of 100 spikes was averaged, aligned to the time-point of the peak of the waveform 
from the channel with greatest amplitude. Since at the time of this recording only 16 EEG channels were available in the magnet, no inverse solutions could be calculated with these data. After verifying similar field topographies of the two recordings, the 29 channel EEG recorded during the EEG video monitoring was used for 3D analysis instead. A general linear inverse solution called 'low resolution electromagnetic tomography' (LORETA; Pascual-Marqui et al., 1994) was used. This method estimates the current density distribution within the whole 3D space, and thus can account for distributed sources without a priori constraints. A 3 shell spherical head model was used for the calculation.

\section{Results}

We were able to record excellent EEG within the magnet, allowing easy detection of spike activity to trigger fMRI acquisition (Fig. 1). On the basis of the applied restrictions outlined above, the EEG-triggered fMRI revealed 3 clusters of BOLD signal increases compared with the control condition: in the left frontal region, the interhemispheric region and in the right frontal lobe (Fig. 2). The 3D current density estimation around the peak of the averaged spikes resulted in a homogeneous temporal succession of the activity, beginning over the left parasagittal frontal structures, followed by propagation over the interhemispheric region to the right frontal lobe (Fig. 3).

The MRI, previously reported as normal, was reviewed by one of us (J.D.) and revealed a small region of thickened cortex which was concordant with the left frontal cluster of pixels (Fig. 2a) and the findings of subdural recordings. In the electrodes overlying the area of signal enhancement, ictal onset and interictal discharges anomalies were noted. Functional mapping with electrical stimulation of the left frontal cortex resulted in speech arrest just close to the epileptogenic focus and partially above the region of thickened cortex. Because of this partial overlap with the 'speech area', no complete resection could be carried out.

Histopathological examination revealed focal gliotic changes. During a follow-up period of 6 months (under

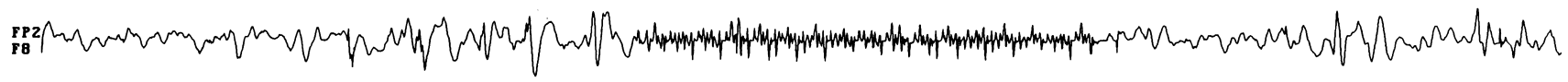

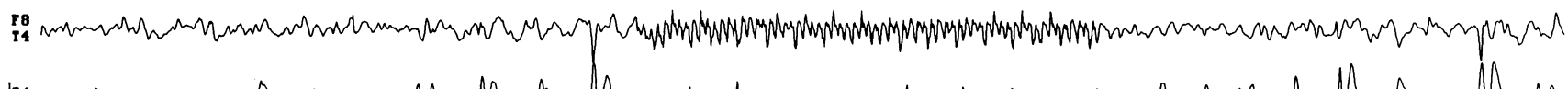

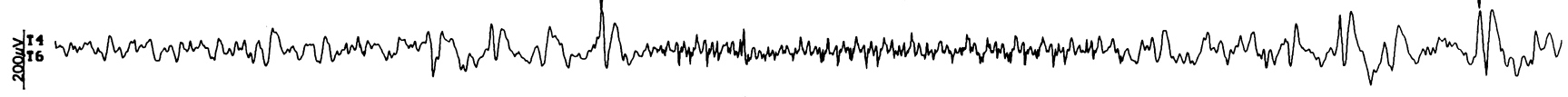

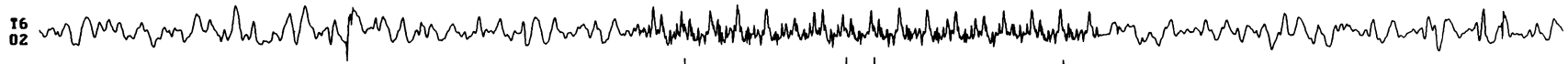

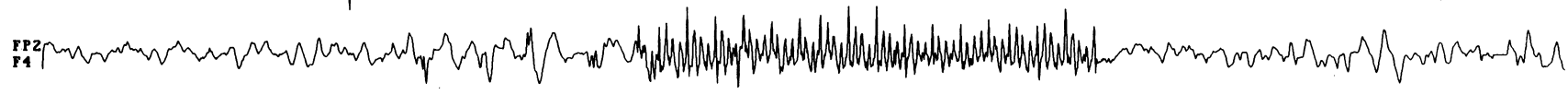

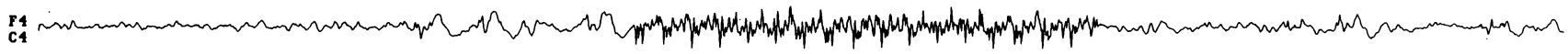

$$
\begin{aligned}
& \text { P4 }
\end{aligned}
$$

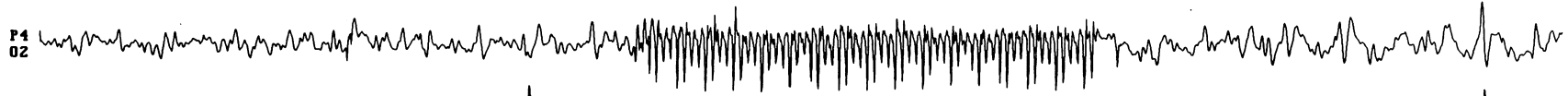

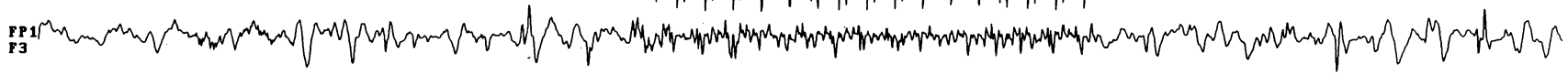

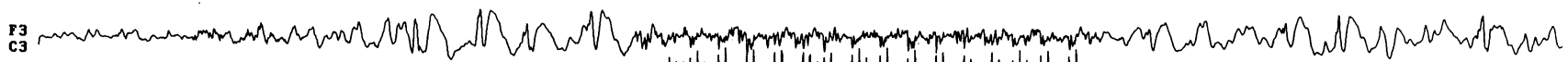

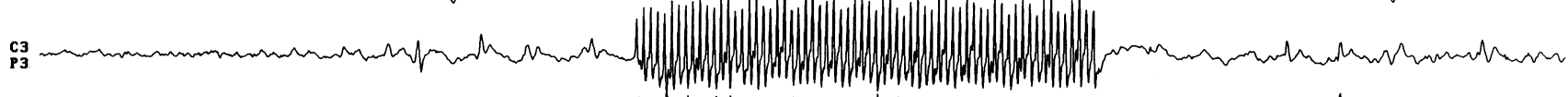

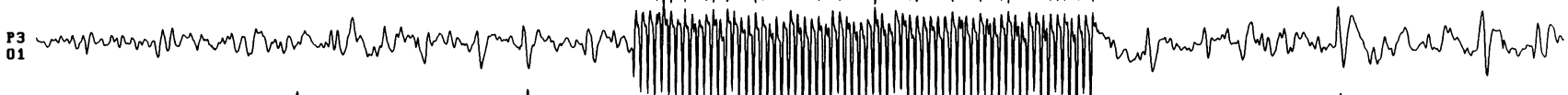

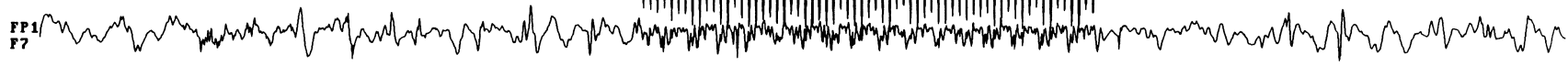

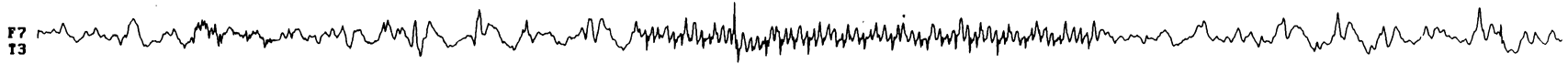

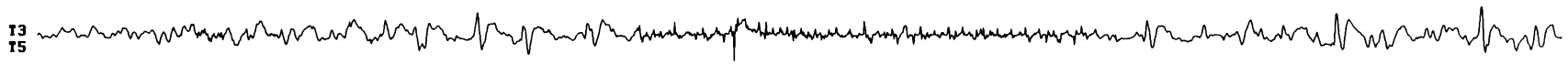

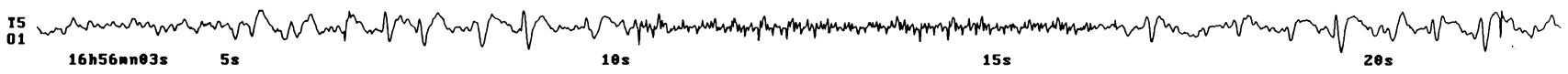

Fig. 1. Example of an EEG with MRI-artifact. Please note the good quality of the EEG before and after MRI-acquisition, allowing detection of the epileptiform discharges. Time scale (real time) on the bottom. h, hour; mn, minute; s, second. 

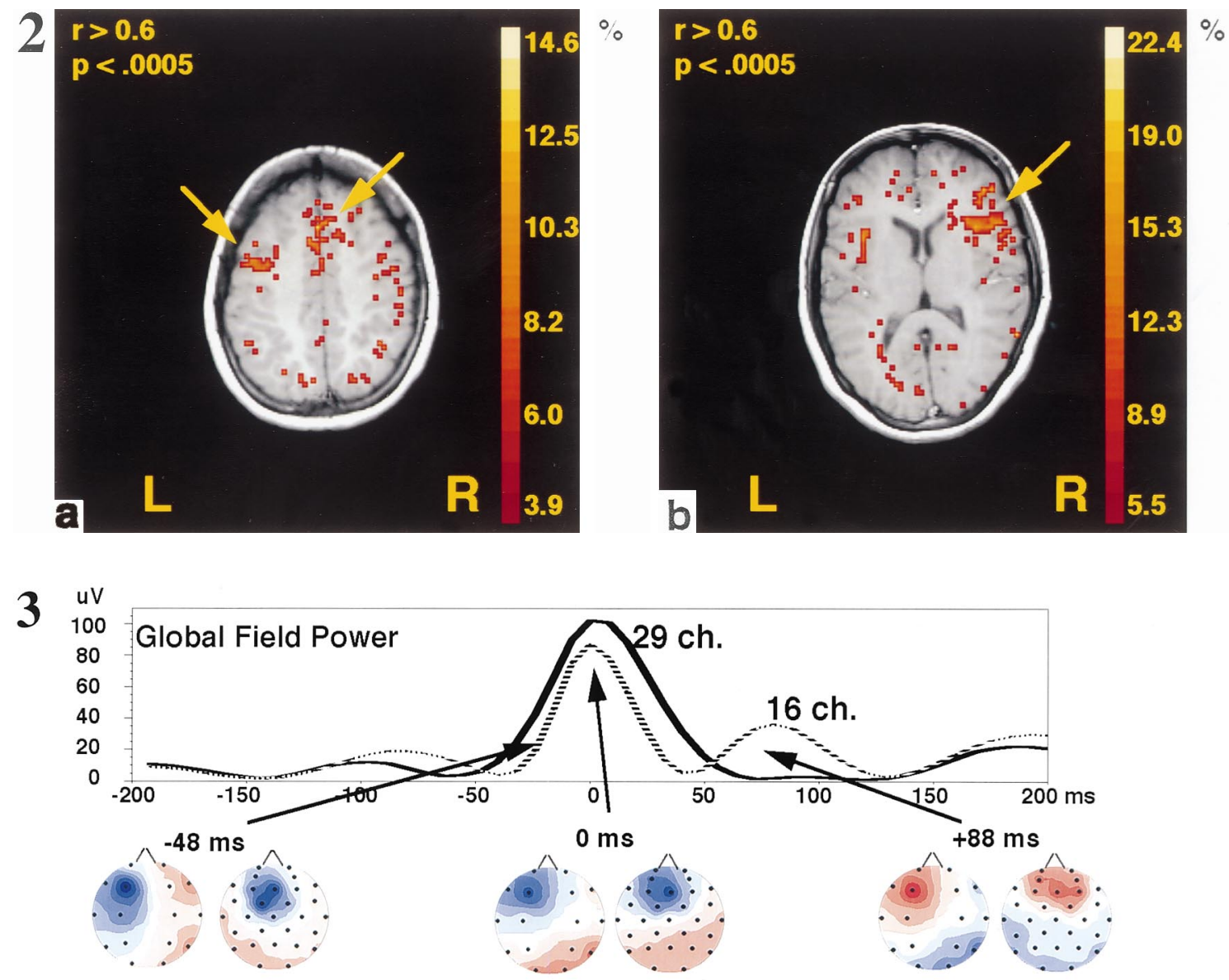

$16 \mathrm{ch}$.

$29 \mathrm{ch}$.
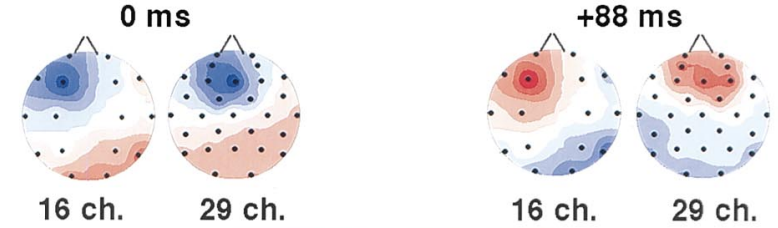

Surface Potential Maps

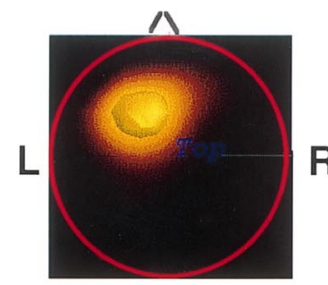

3D-Current Density Distribution (29 channels)
$\mathbf{R}$

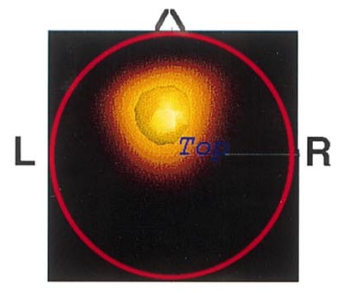

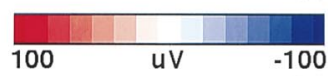

$16 \mathrm{ch}$.

$29 \mathrm{ch}$.

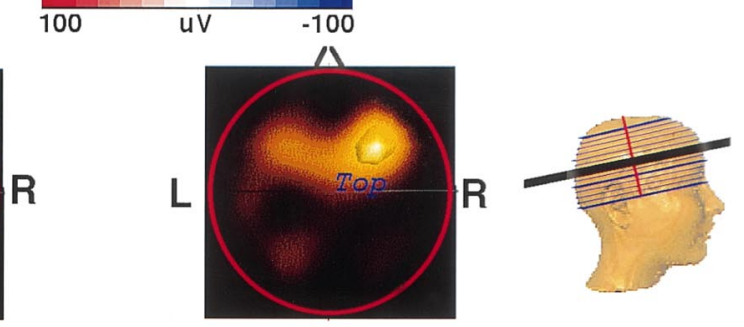

Min. Current Density Max.

Fig. 2. (a,b) fMRI results superimposed on T1-weighted MR images. The color scale represents the percentage changes of BOLD signal intensity between the EEG-triggered and control images. Pixels were selected based on a correlation threshold of 0.6 (Bonferroni corrected $P$-value $<0.005$ ). (a) Two-pixel clusters were found in the left dorsolateral frontal region (arrow) and the mesial regions (arrow). (b) Pixel cluster corresponding to right lateral frontal tissue (arrow).

Fig. 3. Top: waveform of the field strength (global field power) of the averaged epileptic discharges in the 16 and the 29 channel recordings. Middle: surface potential maps are shown for the beginning, the peak and the end of the global field power of the average. Please note the similar topography surface maps of the two recordings. Bottom: 3D-localization using a linear inverse solution for the 29 channel recordings at the same 3 time points. For clarification purposes, the solution is shown on one slice only, located approximately $4 \mathrm{~cm}$ above the T3-T4-Fpz plane. Electric activity starts in the left anterior region and propagates via the midline to the right frontal region.

oxcarbamazepin monotherapy), 5 habitual seizures outside the direct post-operative period occurred, corresponding so far to a marked reduction of her seizure frequency compared with the pre-operative period.

\section{Discussion}

It is well known that patients with extratemporal epilepsy but without MRI-identifiable lesions, who undergo surgery, 
have a chance of only 30-40\% of becoming completely seizure-free post-operatively (Engel, 1989). In this group, scalp recordings rarely allow a precise identification of the epileptogenic zone and usually intracranial EEG recordings, with all their risks, are required. However, even with intracranial depth or subdural electrode recordings, an inherent problem of spatial undersampling remains, which is probably the major determinant for poor post-operative outcome (Quesney et al., 1992).

Here we present a method which seems to be a promising tool for non-invasive identification of seizure foci. In the patient with non-lesional extratemporal epilepsy, the analysis of the EEG-triggered fMRI revealed multiple regions of blood-flow changes in left, mesial and right frontal areas. The 3D analysis of the EEG unfolded the temporal evolution of the pathological electrical activity, i.e. the same 3 regions were identified, but with an onset in the left frontal lobe. The localization of the epileptogenic focus was confirmed by intracranial recordings and histopathological analysis.

Lobar structures, such as the frontal lobe, are complex and anatomically and functionally heterogeneous systems. The precise outline of the epileptogenic region is mandatory, if one plans to resect epileptogenic tissue with maximal chances of post-operative success. Combining the spatial accuracy of EEG-triggered fMRI acquisition with the temporal accuracy of EEG-based electromagnetic tomography aids in the exact localization of the epileptogenic focus. Not only adult, but also pediatric patients would benefit from such a non-invasive and precise technique, in particular if invasive EEG recordings are considered.

\section{Acknowledgements}

We thank Dr. D. L. Schomer for helpful discussions and N. Mainwaring, L. Spinnelli and Y. Ducommun-dit-Boudry for technical support. This work was partially supported by the Swiss National Science Foundation (No. 31-52933.97 and No. 4038-044081).

\section{References}

Bandettini, P.A., Jesmanowicz, A., Wang, E.C. and Hyde, J.S. Processing strategies for time-course data sets in functional MRI of the human brain. Magn. Reson. Med., 1993, 30: 161-173.

Belliveau, J.W., Kennedy, D.N. Jr., McKinstry, R.C., Buchbinder, B.R.,
Weisskoff, R.M., Cohen, M.S., Vevea, J.M., Brady, T.J. and Rosen, B.R. Functional mapping of the human visual cortex. Science, 1991, 254: 716-719.

Detre, J.A., Sirven, J.I., Alsop, D.C., O'Connor, M.J. and French, J.A. Localization of subclinical ictal activity by functional magnetic resonance imaging: correlation with invasive monitoring. Ann. Neurol., 1995, 38: 618-624.

Ebersole, J.S. EEG dipole modelling in complex partial epilepsy. Brain Topogr., 1991, 4: 113-123.

Engel, J., Jr. Seizures and Epilepsy. F.A. Davis, Philadelphia, PA, 1989.

George, J.S., Aine, C.J., Mosher, C., Schmidt, D.M., Ranken, D.M., Schlitt, H.A., Wood, C.C., Lewine, J.D., Sanders, J.A. and Belliveau, J.W. Mapping function in the human brain with magnetoencephalography, anatomical magnetic resonance imaging, and functional magnetic resonance imaging. J. Clin. Neurophysiol., 1995, 12: 406-431.

Henry, T.R., Chugani, H.T., Abou-Khalil, B.W., Theodore, W.H. and Swartz, B.E. Positron emission tomography. In: J. Engel, Jr. (Ed.), Surgical Treatment of the Epilepsies, 2nd edn., Raven Press, New York, 1993, pp. 211-232.

Ives, J.R., Warach, S., Schmitt, F., Edelman, R.R. and Schomer, D.L. Monitoring the patient's EEG during echo planar MRI. Electroenceph. clin. Neurophysiol., 1993, 87: 417-420.

Jackson, G.D., Connelly, A., Cross, J.H., Gordon, I. and Gadian, D.G. Functional magnetic resonance imaging of focal seizures. Neurology, 1994, 44: 850-856.

Jiang, A., Kennedy, D.N., Baker, J.R., Weisskoff, R.M., Tootell, R.B.H., Woods, R.P., Benson, R.R., Brady, T.J., Rosen, B.R. and Belliveau, J.W. Motion detection and correction in functional MR imaging. Hum. Brain Mapp., 1995, 3: 224-235.

Lantz, G., Holub, M., Rydgen, E. and Rosén, I. Simultaneous intracranial and extracranial recording of interictal epileptiform activity in patients with drug resistant partial epilepsy: patterns of conduction and results from dipole reconstruction. Electroenceph. clin. Neurophysiol., 1996, 99: 69-78.

Lantz, G., Michel, C.M., Pascual-Marqui, R.D., Spinelli, L., Seeck, M., Seri, S., Landis, T. and Rosén, I. Extracranial localisation of intracranial interictal epileptiform activity using LORETA (low resolution electromagnetic tomography). Electroenceph. clin. Neurophysiol., 1997, 102: 414-422.

Marks, D.A., Katz, A., Hoffer, P. and Spencer, S.S. Localization of extratemporal epileptic foci during ictal single photon emission computed tomography. Ann. Neurol., 1992, 31: 250-255.

Newton, M.R., Berkovic, S.F., Austin, M.C., Rowe, C.C., McKay, W.J. and Bladin, P.F. SPECT in the localisation of extratemporal and temporal seizure foci. J. Neurol. Neurosurg. Psychiatry, 1995, 59: 26-30.

Pascual-Marqui, R.D., Michel, C.M. and Lehmann, D. Low resolution electromagnetic tomography: a new method to localize electrical activity in the brain. Int. J. Psychophysiol., 1994, 18: 49-65.

Quesney, L.F., Constain, M., Rasmussent, T., Stefan, H. and Olivier, A. How large are frontal lobe epileptogenic zones? Adv. Neurol., 1992, 57 : 311-323.

Seri, S., Cerquiglini, A., Pisani, F., Michel, C.M, Pascual-Marqui, R.D. and Curatolo, P. Frontal lobe epilepsy associated with tuberous sclerosis: EEG-MRI fusioning. J. Child Neurol., 1998, 13: 33-38.

Warach, S., Ives, J.R., Schlaug, G., Patel, M.R., Darby, D.G., Thangaraj, V., Edelman, R.R. and Schomer, D.L. EEG-triggered echo-planar functional MRI in epilepsy. Neurology, 1996, 47: 89-93. 